\title{
BOUNDARY INTEGRAL EQUATIONS FOR AN ANISOTROPIC BIMATERIAL WITH THERMALLY IMPERFECT INTERFACE AND INTERNAL INHOMOGENEITIES
}

\author{
Heorhiy SULYM*, laroslav PASTERNAK", Mykhailo TOMASHIVSKYY**t \\ "Bialystok University of Technology, ul. Wiejska 45C, 15-351 Bialystok, Poland \\ *"Lutsk National Technical University, Lvivska Str. 75, 43018 Lutsk, Ukraine \\ ${ }^{* * *}$ Ivan Franko National University of Lviv, Universytetska Str. 1, 79000 Lviv, Ukraine \\ h.sulym@pb.edu.pl, yaroslav.pasternak@gmail.com, tomashmyh@gmail.com
}

received 4 May 2015, revised 25 February 2016, accepted 26 February 2016

\begin{abstract}
This paper studies a thermoelastic anisotropic bimaterial with thermally imperfect interface and internal inhomogeneities. Based on the complex variable calculus and the extended Stroh formalism a new approach is proposed for obtaining the Somigliana type integral formulae and corresponding boundary integral equations for a thermoelastic bimaterial consisting of two half-spaces with different thermal and mechanical properties. The half-spaces are bonded together with mechanically perfect and thermally imperfect interface, which model interfacial adhesive layers present in bimaterial solids. Obtained integral equations are introduced into the modified boundary element method that allows solving arbitrary 2D thermoelacticity problems for anisotropic bimaterial solids with imperfect thin thermo-resistant interfacial layer, which half-spaces contain cracks and thin inclusions. Presented numerical examples show the effect of thermal resistance of the bimaterial interface on the stress intensity factors at thin inhomogeneities.
\end{abstract}

Key words: Bimaterial, Imperfect Interface, Thermoelastic, Anisotropic, Crack, Thin Inclusion

\section{INTRODUCTION}

Bimaterial parts and structures with different thermal and mechanical properties of their components are widely used in modern engineering design due to their useful anisotropic properties, which thus operate different way in different directions. As a result of such bondage of different materials a thin interfacial layer with finite thickness appears at the interface, which affects the temperature and stress fields in the obtained bimaterial solid. In modeling of the effect of this layer, due to small thickness of the latter it can be reduced to consideration of certain boundary conditions of imperfect interface at the surface of material bondage. However, besides the imperfect interface, another inhomogeneities (such as cracks, thin inclusions etc.) are usually present in structural materials. Therefore, in the study of bimaterial solids one should account for both. Thus, the development of effective methods for modeling of thermal and mechanical fields' distribution in bimaterial solids with imperfect interface and internal thin inhomogeneities is an important practical problem.

Development of efficient techniques for analysis of thermoelasticity problems is very important in the study of contact and friction problems, especially those accounting for thermal emission. Polish scientists vastly develop these studies. Those are Z. Baczyński, J. Ignaczak, A. Kaczyński, M. Kuciej, S.J. Matysiak, V. Pauk, E. Wierzbicki, A. Yevtushenko (e.g. see the recent review by Yevtushenko and Kuciej (2012) and monograph Jewtusheko et al (2014)).

The study of bimaterial solids is widely covered in scientific literature (Benveniste, 2006, Kattis and Mavroyannis, 2006, Pan and Amadei, 1999, Pasternak et al., 2014, Qin, 2007, Wang and
Pan, 2010). In particular, Pan and Amadei (1999) developed an efficient boundary element approach for defective elastic anisotropic bimaterial solids. Hwu (1992) obtained analytic solutions for interfacial cracks in thermoelastic anisotropic dissimilar media. Wang and Pan (2010) derived thermoelastic Green's functions for anisotropic thermoelastic bimaterial with Kapitza-type and spring-type imperfect interface. Pasternak et al. (2014) obtained truly boundary integral equations for 2D thermoelectroelasticity of a defective bimaterial solid with a perfect interface.

However, the thermoelasticity of anisotropic bimaterial solids with imperfect interface containing internal inhomogeneities is less studied. In general, there are mainly two types of imperfect interfaces in the context of heat conduction (Benveniste, 2006, Chen, 2001, Kattis and Mavroyannis, 2006), namely the weakly conducting interface (or the well known Kapitza thermal contact resistance model) and the highly conducting interface. At a weakly conducting interface it is assumed that the normal heat flux is continuous across the interface, and the temperature possesses an interfacial discontinuity, which is proportional to the normal heat flux (Wang and Pan, 2010).

Therefore, this paper studies the thermoelastic anisotropic bimaterial with thermally imperfect and mechanically perfect interface. It is assumed the weakly temperature conducting interface, as in Ref (Wang and Pan, 2010), and tractions and displacements are assumed to be continuous across the interface (perfect mechanical contact). The general complex variable approach of Pasternak et al. (2014) is used to derive closed-form boundary integral formulae and equations for a bimaterial solid with thermally imperfect interface. 


\section{GOVERNING EQUATIONS OF PLANE ANISOTROPIC THERMOELASTICITY AND THE EXTENDED STROH FORMALISM}

In a fixed rectangular system of coordinates $O x_{1} x_{2} x_{3}$ the equilibrium, heat balance, and constitutive equations of plane (in a plane $O x_{1} x_{2}$ ) strain and plane heat conduction for a linearly thermoelastic anisotropic solid can be written as (Hwu, 2010, Pasternak, 2012, Ting, 1996):

$\sigma_{i j, j}=0, h_{i, i}=0(i, j=1,2,3)$;

$\sigma_{i j}=C_{i j k m} \varepsilon_{k m}-\beta_{i j} \theta, h_{i}=-k_{i j} \theta_{, j}$,

where: $\varepsilon_{i j}=\left(u_{i, j}+u_{j, i}\right) / 2$ is a strain tensor; $\sigma_{i j}$ is a stress tensor; $h_{i}$ is a heat flux; $u_{i}$ is a displacement vector; $\theta$ is a temperature change with respect to the reference one; $C_{i j k m}$ are elastic moduli; $k_{i j}$ are heat conduction coefficients; $\beta_{i j}=$ $C_{i j k m} \alpha_{k m}(i, j, k, m=1, \ldots, 3)$ are thermal moduli; $\alpha_{i j}$ are thermal expansion coefficients. Tensors $C_{i j k m}, k_{i j}, \alpha_{i j}$, and $\beta_{i j}$ are fully symmetric. Here and further, the Einstein summation convention is used. A comma at subscript denotes differentiation with respect to a coordinate indexed after the comma, i.e. $u_{i, j}=\frac{\partial u_{i}}{\partial x_{j}}$.

According to the extended Stroh formalism (Hwu, 2010, Ting, 1996) the general homogeneous solution of Eqs (1), (2) writes as:

$\theta=2 \operatorname{Re}\left\{g^{\prime}\left(z_{t}\right)\right\}, \vartheta=2 k_{t} \operatorname{Im}\left\{g^{\prime}\left(z_{t}\right)\right\}$

$h_{1}=-\vartheta_{, 2}, h_{2}=\vartheta_{, 1}, k_{t}=\sqrt{k_{11} k_{22}-k_{12}{ }^{2}}$,

$\mathbf{u}=2 \operatorname{Re}\left[\operatorname{Af}\left(z_{*}\right)+\mathbf{c} g\left(z_{t}\right)\right]$,

$\phi=2 \operatorname{Re}\left[\mathbf{B f}\left(z_{*}\right)+\mathbf{d} g\left(z_{t}\right)\right]$,

$\sigma_{i 1}=-\varphi_{i, 2}, \sigma_{i 2}=\varphi_{i, 1}$,

$z_{t}=x_{1}+p_{t} x_{2}, z_{\alpha}=x_{1}+p_{\alpha} x_{2}$,

$\mathbf{f}\left(z_{*}\right)=\left[F_{1}\left(z_{1}\right), F_{2}\left(z_{2}\right), F_{3}\left(z_{3}\right)\right]^{\mathrm{T}}$,

where: $\vartheta$ is a heat flux function; $F_{\alpha}\left(z_{\alpha}\right)$ and $g\left(z_{t}\right)$ are complex analytic functions with respect to their arguments; the complex constant $p_{t}$ is a root (with a positive imaginary part) of the characteristic equation for heat conduction $k_{22} p_{t}^{2}+2 k_{12} p_{t}+k_{11}=$ 0 . Constant complex matrices $\mathbf{A}, \mathbf{B}$, vectors $\mathbf{c}, \mathbf{d}$, and scalars $p_{\alpha}$ $(\alpha=1,2,3)$ are determined from the extended Stroh eigenvalue problem (Ting, 1996).

Vector $f\left(z_{*}\right)$ of Stroh complex functions is related to the realvalued stress and displacement functions as (Pasternak, 2012):

$\mathbf{f}\left(z_{*}\right)=\mathbf{B}^{\mathrm{T}} \mathbf{u}+\mathbf{A}^{\mathrm{T}} \phi-\mathbf{B}^{\mathrm{T}} \mathbf{u}^{t}-\mathbf{A}^{\mathrm{T}} \phi^{t}$,

$\mathbf{u}^{t}=2 \operatorname{Re}\left\{\mathbf{c} g\left(z_{t}\right)\right\}, \phi^{t}=2 \operatorname{Re}\left\{\mathbf{d} g\left(z_{t}\right)\right\}$.

According to $\mathrm{Eq}(3)$, the Stroh temperature function $g^{\prime}\left(z_{t}\right)$ is related to the heat flux and temperature functions as:

$g^{\prime}\left(z_{t}\right)=\frac{1}{2}\left(\theta+i \frac{\vartheta}{k_{t}}\right)$.

\section{DERIVATION OF THE INTEGRAL FORMULAE FOR A BIMATERIAL SOLID}

Consider plane strain of a medium consisting of two thermoelastic anisotropic half-spaces $S_{1}\left(x_{2}>0\right)$ and $S_{2}\left(x_{2}<0\right)$ (see Fig. 1). Along the line $x_{2}=0$ (actually, the plane $O x_{2} x_{3}$ ), which is a bondage line, the conditions of imperfect thermal contact are satisfied:

$$
\begin{aligned}
& \left.\theta^{(1)}\left(x_{1}, x_{2}\right)\right|_{x_{2}=0}= \\
& \left.\theta^{(2)}\left(x_{1}, x_{2}\right)\right|_{x_{2}=0}-\left.\rho_{0}\left(\vartheta_{, 1}^{(2)}\left(x_{1}, x_{2}\right)\right)\right|_{x_{2}=0}, \\
& \left.\vartheta^{(1)}\left(x_{1}, x_{2}\right)\right|_{x_{2}=0}=\left.\vartheta^{(2)}\left(x_{1}, x_{2}\right)\right|_{x_{2}=0},
\end{aligned}
$$

and perfect mechanical contact holds

$$
\begin{aligned}
& \left.\phi^{(1)}\left(x_{1}, x_{2}\right)\right|_{x_{2}=0}=\left.\phi^{(2)}\left(x_{1}, x_{2}\right)\right|_{x_{2}=0}, \\
& \left.\mathbf{u}^{(1)}\left(x_{1}, x_{2}\right)\right|_{x_{2}=0}=\left.\mathbf{u}^{(2)}\left(x_{1}, x_{2}\right)\right|_{x_{2}=0} .
\end{aligned}
$$

Here and further superscripts 1 or 2 denote corresponding half-space $S_{1}$ or $S_{2}$, respectively, which the field quantity belongs to. Boundary conditions (6) and (7) from the physical point of view correspond to a model of a thin layer, for which according to Fourier law of heat conduction the temperature difference at the bimaterial interface is proportional to the heat flux through it. Thus, the parameter $\rho_{0}$ in Eq (6) is a thermal resistance of the abovementioned layer, and as a consequence, a thermal resistance of the bimaterial interface. As $\rho_{0} \rightarrow 0$ or $\rho_{0} \rightarrow \infty$ one obtains perfect thermal contact or thermally insulated bimaterial interface (adiabatic contact), respectively.

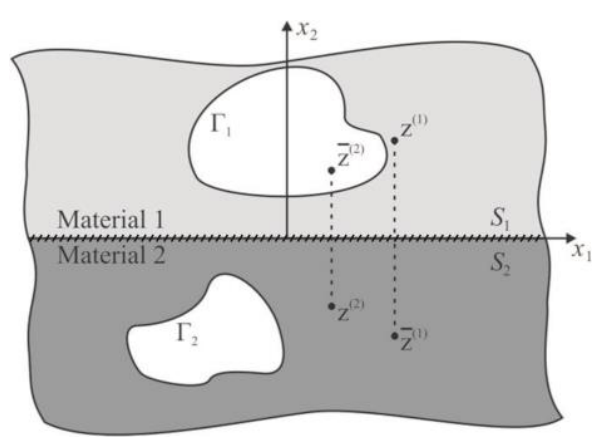

Fig. 1. Thermoelastic anisotropic bimaterial medium

It is considered that each of the half-spaces contains systems of cylindrical holes, which are represented with plane contours $\Gamma_{1}=\mathrm{U}_{i} \Gamma_{i}^{(1)}$ and $\Gamma_{2}=\mathrm{U}_{i} \Gamma_{i}^{(2)}$, respectively.

For derivation of the integral formulae for the Stroh complex functions for bonded half-spaces one can use the Cauchy integral formula (Muskhelishvili, 2008), which relates values of an arbitrary analytic function $\phi(\tau)$ at the boundary $\partial S$ of the domain $S$ with its value inside this domain:

$\frac{1}{2 \pi i} \int_{\partial S} \frac{\phi(\tau) d \tau}{\tau-z}= \begin{cases}\phi(z) & \forall z \in S, \\ 0 & \forall z \notin S,\end{cases}$

where $\tau, z \in \mathbb{C}$ are complex variables, which define the position of the source and field points, respectively. Herewith, if the domain $S$ is infinite it is assumed that the function $\phi(z)$ vanishes at $z \rightarrow \infty$.

\subsection{Heat conduction}

Due to the linearity of the problem of heat conduction one can present its solution as a superposition of the homogeneous solu- 
tion given by the functions $g_{1 \infty}\left(z_{t}^{(1)}\right)$ and $g_{2 \infty}\left(z_{t}^{(2)}\right)$ (which should definitely satisfy the boundary conditions (6)), and the perturbed solution caused by the presence of the contours $\Gamma_{1}$ and $\Gamma_{2}$.

Denote the Cauchy integrals of the complex temperature functions $g_{i}^{\prime}\left(z_{t}^{(i)}\right)$ as:

$q_{t}^{(i)}\left(z_{t}^{(j)}\right)=\int_{\Gamma_{i}} \frac{g_{i}^{\prime}\left(\tau_{t}^{(i)}\right) d \tau_{t}^{(i)}}{\tau_{t}^{(i)}-z_{t}^{(j)}}$,

$\bar{q}_{t}^{(i)}\left(z_{t}^{(j)}\right)=\int_{\Gamma_{i}} \frac{\overline{g_{t}^{\prime}\left(\tau_{t}^{(i)}\right)} d \tau_{t}^{(i)}}{\bar{\tau}_{t}^{(i)}-z_{t}^{(j)}}$

and the improper integrals over the infinite path $-\infty<x_{1}<$ $+\infty$ as

$m_{t}\left(z_{t}^{(j)}\right)=\int_{-\infty}^{+\infty} \frac{\vartheta\left(x_{1}\right) d x_{1}}{x_{1}-z_{t}^{(j)}}$,

$p_{t}\left(z_{t}^{(j)}\right)=\int_{-\infty}^{+\infty} \frac{\theta^{(2)}\left(x_{1}\right) d x_{1}}{x_{1}-z_{t}^{(j)}}$.

Integration by parts of the improper integral gives:

$\int_{-\infty}^{+\infty} \frac{\vartheta, 1\left(x_{1}\right)}{x_{1}-z_{t}^{(j)}} d x_{1}=\left.\frac{\vartheta\left(x_{1}\right)}{x_{1}-z_{t}^{(j)}}\right|_{-\infty} ^{+\infty}+$

$\int_{-\infty}^{+\infty} \frac{\vartheta\left(x_{1}\right)}{\left(x_{1}-z_{t}^{(j)}\right)^{2}} d x_{1}=m_{t}^{\prime}\left(z_{t}^{(j)}\right)$.

Accounting for the thermal balance conditions, the function $\vartheta\left(x_{1}\right)$ tends to zero at the infinity, therefore the first term in $\mathrm{Eq}$ (12) vanishes, and the second term is a derivative of a function $\mathrm{m}_{\mathrm{t}}^{\prime}\left(\mathrm{z}_{\mathrm{t}}^{(\mathrm{j})}\right)$. Thus,

$\int_{-\infty}^{+\infty} \frac{\vartheta_{1}\left(x_{1}\right)}{x_{1}-z_{t}^{(j)}} d x_{1}=m^{\prime} t\left(z_{t}^{(j)}\right)$.

Utilizing Eqs (5), (9)-(11), (13) and accounting for the boundary conditions at the bimaterial interface the Cauchy integral formulae for the functions $g_{1}^{\prime}\left(z_{t}^{(1)}\right)$ and $g^{\prime}{ }_{2}\left(z_{t}^{(2)}\right)$ write as

$g_{1}^{\prime}\left(z_{t}^{(1)}\right)=g_{1 \infty}^{\prime}\left(z_{t}^{(1)}\right)+\frac{1}{2 \pi i} q_{t}^{(1)}\left(z_{t}^{(1)}\right)+$ $\frac{1}{4 \pi i} p_{t}^{(1)}\left(z_{t}^{(1)}\right)+\frac{1}{4 \pi k_{t}} m_{t}^{(1)}\left(z_{t}^{(1)}\right)-\frac{\rho_{0}}{4 \pi i} m_{t}^{\prime}\left(z_{t}^{(1)}\right)$,

$\forall \operatorname{Im}\left(z_{t}^{(1)}\right)>0$

$g_{2}^{\prime}\left(z_{t}^{(2)}\right)=g_{2 \infty}^{\prime}\left(z_{t}^{(2)}\right)+\frac{1}{2 \pi i} q_{t}^{(2)}\left(z_{t}^{(2)}\right)-$

$\frac{1}{4 \pi i} p_{t}\left(z_{t}^{(2)}\right)-\frac{1}{4 \pi k_{t}^{(2)}} m_{t}\left(z_{t}^{(2)}\right), \forall \operatorname{Im}\left(z_{t}^{(2)}\right)<0$

and for determination of the improper integrals (11) through the Cauchy integrals (10) one obtains the following system of firstorder ordinary differential equations:

$\forall \operatorname{Im}\left(z_{t}^{(1)}\right)>0: q_{t}^{(2)}\left(z_{t}^{(1)}\right)-\frac{1}{2} p_{t}\left(z_{t}^{(1)}\right)-$

$\frac{i}{2 k_{t}^{(2)}} m_{t}\left(z_{t}^{(1)}\right)=0$,

$\bar{q}_{t}^{(1)}\left(z_{t}^{(1)}\right)+\frac{1}{2} p_{t}\left(z_{t}^{(1)}\right)-\frac{i}{2 k_{t}^{(1)}} m_{t}\left(z_{t}^{(1)}\right)-$

$\frac{\rho_{0}}{2} m^{\prime}\left(z_{t}^{(1)}\right)=0$,

$\forall \operatorname{Im}\left(z_{t}^{(2)}\right)<0: \bar{q}_{t}^{(2)}\left(z_{t}^{(2)}\right)-\frac{1}{2} p_{t}\left(z_{t}^{(2)}\right)+$ $\frac{i}{2 k_{t}^{(2)}} m_{t}\left(z_{t}^{(2)}\right)=0$,

$\bar{q}_{t}^{(1)}\left(z_{t}^{(2)}\right)+\frac{1}{2} p_{t}\left(z_{t}^{(2)}\right)+\frac{i}{2 k_{t}^{(1)}} m_{t}\left(z_{t}^{(1)}\right)-$

$\frac{\rho_{0}}{2} m^{\prime}\left(z_{t}^{(2)}\right)=0$.

Satisfying an evident condition that $m_{t}\left(z_{t}^{(j)}\right) \rightarrow 0$ as $z_{t}^{(j)} \rightarrow$ $\infty$ one obtains the solution of systems (15), (16) as:

$p_{t}\left(z_{t}^{(1)}\right)=2 \cdot q_{t}^{(2)}\left(z_{t}^{(1)}\right)+\frac{2 i}{\rho_{0} k_{t}^{(2)}}\left[e_{t}^{(2)}\left(z_{t}^{(1)}\right)+\right.$

$\left.\bar{e}_{t}^{(1)}\left(z_{t}^{(1)}\right)\right]$,

$m_{t}\left(z_{t}^{(1)}\right)=-\frac{2}{\rho_{0}}\left[e_{t}^{(2)}\left(z_{t}^{(1)}\right)+\bar{e}_{t}^{(1)}\left(z_{t}^{(1)}\right)\right]$,

$p_{t}\left(z_{t}^{(2)}\right)=2 \cdot \bar{q}_{t}^{(2)}\left(z_{t}^{(2)}\right)-\frac{2 i}{\rho_{0} k_{t}^{(2)}}\left[e_{t}^{(1)}\left(z_{t}^{(2)}\right)+\right.$

$\left.\bar{e}_{t}^{(2)}\left(z_{t}^{(2)}\right)\right], m_{t}\left(z_{t}^{(2)}\right)=-\frac{2}{\rho_{0}}\left[e_{t}^{(1)}\left(z_{t}^{(2)}\right)+\right.$

$\left.\bar{e}_{t}^{(2)}\left(z_{t}^{(2)}\right)\right]$.

New functions $e_{t}^{(i)}\left(z_{t}^{(j)}\right)$ and $\bar{e}_{t}^{(i)}\left(z_{t}^{(j)}\right)$ are defined though the following integral formulae

$e_{t}^{(i)}\left(z_{t}^{(j)}\right)=\int_{\Gamma_{i}} g^{\prime}{ }_{i}\left(\tau_{t}^{(i)}\right) \mathrm{K}\left(B^{(j)}\left(\tau_{t}^{(i)}-z_{t}^{(j)}\right)\right) d \tau_{t}^{(i)}$,

$e_{t}^{(i)}\left(z_{t}^{(j)}\right)=\int_{\Gamma_{i}} g^{\prime}{ }_{i}\left(\tau_{t}^{(i)}\right) \mathrm{K}\left(B^{(j)}\left(\tau_{t}^{(i)}-z_{t}^{(j)}\right)\right) d \tau_{t}^{(i)}$,

where: $\mathrm{K}(z)=e^{z} E_{1}(z)$,

$B^{(1)}=\frac{i}{\rho_{0}} \frac{k_{t}^{(1)}+k_{t}^{(2)}}{k_{t}^{(1)} \cdot k_{t}^{(2)}}$,

$B^{(2)}=-\frac{i}{\rho_{0}} \frac{k_{t}^{(1)}+k_{t}^{(2)}}{k_{t}^{(1)} \cdot k_{t}^{(2)}}$

and $E_{1}(z)$ is an exponential integral defined as

$E_{1}(z)=\int_{z}^{\infty} \frac{e^{-t}}{t} d t$.

Substituting obtained solution into $\mathrm{Eq}$ (14) one can derive the integral formulae for the Stroh complex temperature functions $g_{1}^{\prime}\left(z_{t}^{(1)}\right)$ and $g^{\prime}{ }_{2}\left(z_{t}^{(2)}\right)$, which do not contain the improper integrals over the infinite bimaterial interface:

$$
\begin{aligned}
& \forall \operatorname{Im}\left(z_{t}^{(1)}\right)>0: g^{\prime}\left(z_{t}^{(1)}\right)=g^{\prime}{ }_{1 \infty}\left(z_{t}^{(1)}\right)+ \\
& \frac{1}{2 \pi i}\left[q_{t}^{(1)}\left(z_{t}^{(1)}\right)-\bar{q}_{t}^{(1)}\left(z_{t}^{(1)}\right)+\frac{2 i}{\rho_{0} k_{t}^{(1)}}\left(e_{t}^{(2)}\left(z_{t}^{(1)}\right)+\right.\right. \\
& \left.\left.\bar{e}_{t}^{(1)}\left(z_{t}^{(1)}\right)\right)\right], \\
& \forall \operatorname{Im}\left(z_{t}^{(2)}\right)>0: g_{2}^{\prime}\left(z_{t}^{(2)}\right)=g^{\prime}{ }_{2 \infty}\left(z_{t}^{(2)}\right)+ \\
& \frac{1}{2 \pi i}\left[q_{t}^{(2)}\left(z_{t}^{(2)}\right)-\bar{q}_{t}^{(2)}\left(z_{t}^{(2)}\right)-\frac{2 i}{\rho_{0} k_{t}^{(2)}}\left(e_{t}^{(1)}\left(z_{t}^{(2)}\right)+\right.\right. \\
& \left.\left.\bar{e}_{t}^{(2)}\left(z_{t}^{(2)}\right)\right)\right] .
\end{aligned}
$$

Using (5) one can derive the integral formulae for the functions $g_{1}^{\prime}\left(z_{t}^{(1)}\right)$ and $g_{2}^{\prime}\left(z_{t}^{(2)}\right)$ relating them with the boundary values of temperature $\theta$ and normal component $h_{n}=h_{i} n_{i}$ of a heat flux vector $\left(n_{i}\right.$ is a unit outwards normal vector to a curve $\Gamma_{i}$ at its certain point).

According to Eqs (5), (23), and (24), one can obtain the inte- 
gral formulae for temperature and heat flux at the arbitrary point $\xi$ of a bimaterial solid:

$\theta(\xi)=\left\{\begin{array}{l}2 \operatorname{Re}\left\{g^{\prime}{ }_{1}\left(Z_{t}^{(1)}(\xi)\right)\right\}\left(\forall \xi \in S_{1}\right), \\ 2 \operatorname{Re}\left\{g^{\prime}{ }_{2}\left(Z_{t}^{(2)}(\xi)\right)\right\}\left(\forall \xi \in S_{2}\right),\end{array}=\right.$

$\int_{\Gamma}\left[\Theta^{\mathrm{ITC} *}(\mathbf{x}, \xi) h_{n}(\mathbf{x})-H^{\mathrm{ITC} *}(\mathbf{x}, \xi) \theta(\mathbf{x})\right] d s(x)+$ $\theta^{\infty}(\xi)$

$h_{i}(\xi)=$

$\left\{\begin{array}{l}2 k_{t}^{(1)} \operatorname{Im}\left\{\left(\delta_{2 i}-\delta_{1 i} p_{t}^{(1)}\right) g^{\prime \prime}{ }_{1}\left(Z_{t}^{(1)}(\xi)\right)\right\}\left(\forall \xi \in S_{1}\right), \\ 2 k_{t}^{(2)} \operatorname{Im}\left\{\left(\delta_{2 i}-\delta_{1 i} p_{t}^{(2)}\right) g^{\prime \prime}{ }_{2}\left(Z_{t}^{(2)}(\xi)\right)\right\}\left(\forall \xi \in S_{2}\right),\end{array}=\right.$

$\int_{\Gamma}\left[\Theta_{i}^{\mathrm{ITC} * *}(\mathbf{x}, \xi) h_{n}(\mathbf{x})-H_{i}^{\mathrm{ITC} * *}(\mathbf{x}, \xi) \theta(\mathbf{x})\right] d s(\mathbf{x})+$ $h_{i}^{\infty}(\xi)$,

where: the functions $\theta^{\infty}(\xi)$ and $h_{i}^{\infty}(\xi)$ define the homogeneous solution for the unnotched bimaterial

$\theta^{\infty}(\xi)= \begin{cases}2 \operatorname{Re}\left[g^{\prime}{ }_{1 \infty}\left(Z_{t}^{(1)}(\xi)\right)\right] & \forall \xi \in S_{1}, \\ 2 \operatorname{Re}\left[g^{\prime}{ }_{2 \infty}\left(Z_{t}^{(2)}(\xi)\right)\right] & \forall \xi \in S_{2},\end{cases}$

$h_{i}^{\infty}(\xi)=$

$\int 2 k_{t}^{(1)} \operatorname{Im}\left[\left(\delta_{2 i}-\delta_{1 i} p_{t}^{(1)}\right) g^{\prime \prime}{ }_{1 \infty}\left(Z_{t}^{(1)}(\xi)\right)\right] \quad \forall \xi \in S_{1}$, $2 k_{t}^{(2)} \operatorname{Im}\left[\left(\delta_{2 i}-\delta_{1 i} p_{t}^{(2)}\right) g^{\prime \prime}{ }_{2 \infty}\left(Z_{t}^{(2)}(\xi)\right)\right] \quad \forall \xi \in S_{2}$.

According to Eqs (3), (23), (24) the kernels of the heat conduction integral formulae are as follows:

$\mathbf{x} \in S_{1} \wedge \xi \in S_{1}:$

$\Theta^{I T C *}(\mathbf{x}, \xi)=\frac{1}{2 \pi k_{t}^{(1)}} \operatorname{Re}\left\{\ln W_{t}^{(1,1)}+\ln \bar{W}_{t}^{(1,1)}\right.$

$\left.+(K-1) L^{(1)}\left(\bar{W}_{t}^{(1,1)}\right)\right\}$,

$H^{I T C *}(\mathbf{x}, \xi)=\frac{1}{2 \pi} \operatorname{Im}\left\{\frac{n_{t}^{(1)}}{W_{t}^{(1,1)}}-\frac{\bar{n}_{t}^{(1)}}{\bar{W}_{t}^{(1,1)}}+\frac{2 i \bar{n}_{t}^{(1)}}{\rho_{0} k_{t}^{(1)}} K\left(B^{(1)} \bar{W}_{t}^{(1,1)}\right)\right\}$,

$\Theta_{i}^{I T C * *}(\mathbf{x}, \xi)=-\frac{1}{2 \pi} \operatorname{Im}\left\{\delta_{t}^{(1)}\left(\frac{1}{W_{t}^{(1,1)}}+\frac{1}{\bar{w}_{t}^{(1,1)}}\right.\right.$

$\left.\left.-\frac{2 i}{\rho_{0} k_{t}^{(1)}} K\left(B^{(1)} \bar{W}_{t}^{(1,1)}\right)\right)\right\}$,

$H_{i}^{I T C * *}(\mathbf{x}, \xi)=-\frac{k_{t}^{(1)}}{2 \pi} \operatorname{Re}\left\{\delta_{t}^{(1)}\left(\frac{n_{t}^{(1)}}{\left(w_{t}^{(1,1)}\right)^{2}}-\frac{\bar{n}_{t}^{(1)}}{\left(\bar{w}_{t}^{(1,1)}\right)^{2}}\right.\right.$

$\left.\left.+\frac{2 i \bar{n}_{t}^{(1)}}{\rho_{0} k_{t}^{(1)}}\left(B^{(1)} K\left(B^{(1)} \bar{W}_{t}^{(1,1)}\right)-\frac{1}{\bar{W}_{t}^{(1,1)}}\right)\right)\right\} ;$

$\mathbf{x} \in S_{2} \wedge \xi \in S_{1}:$

$\Theta^{I T C *}(\mathbf{x}, \xi)=\frac{1-K}{2 \pi k_{t}^{(2)}} \operatorname{Re}\left\{\ln W_{t}^{(2,1)}+\mathrm{K}\left(B^{(1)} W_{t}^{(2,1)}\right)\right\}$,

$H^{I T C *}(\mathbf{x}, \xi)=\frac{1}{\rho_{0} \pi k_{t}^{(1)}} \operatorname{Im}\left\{i n_{t}^{(2)} \mathrm{K}\left(B^{(1)} W_{t}^{(2,1)}\right)\right\}$,

$\Theta_{i}^{I T C * *}(\mathbf{x}, \xi)=-\frac{1}{\rho_{0} \pi k_{t}^{(2)}} \operatorname{Im}\left\{i \delta_{t}^{(1)} \mathrm{K}\left(B^{(1)} W_{t}^{(2,1)}\right)\right\}$

,$H_{i}^{I T C * *}(\mathbf{x}, \xi)=$

$\frac{1}{\rho_{0} \pi} \operatorname{Re}\left\{\delta_{t}^{(1)} n_{t}^{(2)}\left(i B^{(1)} K\left(B^{(1)} W_{t}^{(2,1)}\right)-\frac{1}{W_{t}^{(2,1)}}\right)\right\}$;

$\mathbf{x} \in S_{1} \wedge \xi \in S_{2}$ :

$\Theta^{I T C *}(\mathbf{x}, \xi)=\frac{1+K}{2 \pi k_{t}^{(1)}} \operatorname{Re}\left\{\ln W_{t}^{(1,2)}+K\left(B^{(2)} W_{t}^{(1,2)}\right)\right\}$,

$H^{I T C *}(\mathbf{x}, \xi)=-\frac{1}{\rho_{0} \pi k_{t}^{(2)}} \operatorname{Im}\left\{i n_{t}^{(1)} \mathrm{K}\left(B^{(2)} W_{t}^{(1,2)}\right)\right\}$,
$\Theta_{i}^{I T C * *}(\mathbf{x}, \xi)=\frac{1}{\rho_{0} \pi k_{t}^{(1)}} \operatorname{Im}\left\{i \delta_{t}^{(2)} \mathrm{K}\left(B^{(2)} W_{t}^{(1,2)}\right)\right\}$,

$H_{i}^{I T C * *}(\mathbf{x}, \xi)=$

$-\frac{1}{\rho_{0} \pi} \operatorname{Re}\left\{\delta_{t}^{(2)} n_{t}^{(1)}\left(i B^{(2)} K\left(B^{(2)} W_{t}^{(1,2)}\right)-\frac{1}{W_{t}^{(1,2)}}\right)\right\}$;

$\mathbf{x} \in S_{2} \wedge \xi \in S_{2}$ :

$\Theta^{I T C *}(\mathbf{x}, \xi)=\frac{1}{2 \pi k_{t}^{(2)}} \operatorname{Re}\left\{\ln W_{t}^{(2,2)}+\ln \bar{W}_{t}^{(2,2)}\right.$

$\left.-(K+1) L^{(2)}\left(\bar{W}_{t}^{(2,2)}\right)\right\}$,

$H^{I T C *}(\mathbf{x}, \xi)=\frac{1}{2 \pi} \operatorname{Im}\left\{\frac{n_{t}^{(2)}}{W_{t}^{(2,2)}}-\frac{\bar{n}_{t}^{(2)}}{\bar{w}_{t}^{(2,2)}}-\frac{2 i \bar{n}_{t}^{(2)}}{\rho_{0} k_{t}^{(2)}} K\left(B^{(2)} \bar{W}_{t}^{(2,2)}\right)\right\}$,

$\Theta_{i}^{I T C * *}(\mathbf{x}, \xi)=$

$-\frac{1}{2 \pi} \operatorname{Im}\left\{\delta_{t}^{(2)}\left(\frac{1}{w_{t}^{(2,2)}}+\frac{1}{\bar{w}_{t}^{(2,2)}}+\frac{2 i}{\rho_{0} k_{t}^{(2)}} K\left(B^{(2)} \bar{W}_{t}^{(2,2)}\right)\right)\right\}$

$H_{i}^{I T C * *}(\mathbf{x}, \xi)=\frac{-k_{t}^{(1)}}{2 \pi} \operatorname{Re}\left\{\delta_{t}^{(2)}\left(\frac{n_{t}^{(2)}}{\left(w_{t}^{(2,2)}\right)^{2}}-\frac{\bar{n}_{t}^{(2)}}{\left(\bar{w}_{t}^{(2,2)}\right)^{2}}\right.\right.$

$\left.\left.-\frac{2 i \bar{n}_{t}^{(2)}}{\rho_{0} k_{t}^{(2)}}\left(B^{(2)} K\left(B^{(1)} \bar{W}_{t}^{(2,2)}\right)-\frac{1}{\bar{W}_{t}^{(2,2)}}\right)\right)\right\}$,

where:

$W_{t}^{(i, j)}=Z_{t}^{(i)}(\mathbf{x})-Z_{t}^{(j)}(\xi), \bar{W}_{t}^{(i, j)}=\bar{Z}_{t}^{(i)}(\mathbf{x})-Z_{t}^{(j)}(\xi)$,

$n_{t}^{(i)}=n_{2}(\mathbf{x})-p_{t}^{(i)} n_{1}(\mathbf{x}), \delta_{t}^{(j)}=\delta_{i 2}-p_{t}^{(j)} \delta_{i 1}$,

$\mathrm{L}^{(i)}(z)=\mathrm{K}\left(B^{(i)} z\right)+\ln z$.

According to Eqs (3) and (4), for derivation of integral formulae for extended displacement and extended stress function one should evaluate the anti-derivatives of functions $g_{i}^{\prime}\left(z_{t}^{(i)}\right)$, $m_{t}(z)$, and $p_{t}(z)$ as:

$g_{i}\left(z_{t}^{(j)}\right)=\int g^{\prime}{ }_{i}\left(z_{t}^{(j)}\right) d z_{t}^{(j)}$,

$M_{t}(z)=\int m_{t}(z) d z=-\int_{-\infty}^{+\infty} \ln \left(x_{1}-z\right) \vartheta\left(x_{1}\right) d x_{1}$,

$P_{t}(z)=\int p_{t}(z) d z=-\int_{-\infty}^{+\infty} \ln \left(x_{1}-z\right) \theta\left(x_{1}\right) d x_{1}$.

Utilizing Eqs (23), (24), one obtains:

$\forall \operatorname{Im}\left(z_{t}^{(1)}\right)>0$ :

$g_{1}\left(z_{t}^{(1)}\right)=g_{1 \infty}\left(z_{t}^{(1)}\right)+\frac{1}{2 \pi i}\left[Q_{t}^{(1)}\left(z_{t}^{(1)}\right)-K \bar{Q}_{t}^{(1)}\left(z_{t}^{(1)}\right)\right.$

$\left.+(1-K) Q_{t}^{(2)}\left(z_{t}^{(1)}\right)+(K-1)\left(e_{t}^{(2)}\left(z_{t}^{(1)}\right)+\bar{e}_{t}^{(1)}\left(z_{t}^{(1)}\right)\right)\right]$,

$P_{t}\left(z_{t}^{(1)}\right)=(1-K) Q_{t}^{(2)}\left(z_{t}^{(1)}\right)-(1+K) \bar{Q}_{t}^{(1)}\left(z_{t}^{(1)}\right)$

$+(1+K)\left(e_{t}^{(2)}\left(z_{t}^{(1)}\right)+\bar{e}_{t}^{(1)}\left(z_{t}^{(1)}\right)\right)$,

$M_{t}\left(z_{t}^{(1)}\right)=-2 i k_{t}^{(1)}(1-K)\left[\bar{Q}_{t}^{(1)}\left(z_{t}^{(1)}\right)+Q_{t}^{(2)}\left(z_{t}^{(1)}\right)\right.$

$\left.-\left(e_{t}^{(2)}\left(z_{t}^{(1)}\right)+\bar{e}_{t}^{(1)}\left(z_{t}^{(1)}\right)\right)\right]$;

$\forall \operatorname{Im}\left(z_{t}^{(2)}\right)<0$ :

$g_{2}\left(z_{t}^{(2)}\right)=g_{2 \infty}\left(z_{t}^{(2)}\right)+\frac{1}{2 \pi i}\left[Q_{t}^{(2)}\left(z_{t}^{(2)}\right)+K \bar{Q}_{t}^{(2)}\left(z_{t}^{(2)}\right)\right.$

$\left.+(1+K) Q_{t}^{(1)}\left(z_{t}^{(2)}\right)-(K+1)\left(e_{t}^{(1)}\left(z_{t}^{(2)}\right)+\bar{e}_{t}^{(2)}\left(z_{t}^{(2)}\right)\right)\right]$,

$P_{t}\left(z_{t}^{(2)}\right)=(1-K) \bar{Q}_{t}^{(2)}\left(z_{t}^{(2)}\right)-(1+K) Q_{t}^{(1)}\left(z_{t}^{(2)}\right)$

$+(1+K)\left(e_{t}^{(1)}\left(z_{t}^{(2)}\right)+\bar{e}_{t}^{(2)}\left(z_{t}^{(2)}\right)\right)$, 


$$
\begin{aligned}
& M_{t}\left(z_{t}^{(2)}\right)=2 i k_{t}^{(2)}(1+K)\left[\bar{Q}_{t}^{(2)}\left(z_{t}^{(2)}\right)+Q_{t}^{(1)}\left(z_{t}^{(2)}\right)\right. \\
& \left.-\left(e_{t}^{(1)}\left(z_{t}^{(2)}\right)+\bar{e}_{t}^{(2)}\left(z_{t}^{(2)}\right)\right)\right],
\end{aligned}
$$$$
\int_{-\infty}^{+\infty} \frac{\mathbf{f}^{(j)} d x_{1}}{x_{1}-z_{\beta}^{(i)}}=\mathbf{A}_{j}^{\mathrm{T}} \mathbf{m}\left(z_{\beta}^{(i)}\right)+\mathbf{B}_{j}^{\mathrm{T}} \mathbf{p}\left(z_{\beta}^{(i)}\right)
$$$$
-2 \int_{-\infty}^{+\infty} \frac{\left(\mathbf{A}_{j}^{\mathrm{T}} \operatorname{Re}\left[\mathbf{d}_{j} g_{j}\left(x_{1}\right)\right]+\mathbf{B}_{j}^{\mathrm{T}} \mathrm{Re}\left[\mathbf{c}_{j} g_{j}\left(x_{1}\right)\right]\right) d x_{1}}{x_{1}-z_{\beta}^{(i)}}
$$

where according to Eqs (5) and (28), (29),

$\bar{Q}_{t}^{(i)}(z)=\int \bar{q}_{t}^{(i)}(z) d z=\frac{i}{2 k_{t}^{(i)}} \int_{\Gamma_{i}} f^{*}\left(\bar{\tau}_{t}^{(i)}(s)-z\right) h_{n}(s) d s$

$+\frac{1}{2} \int_{\Gamma_{i}}\left(n_{2}(s)-\bar{p}_{t}^{(i)} n_{1}(s)\right) \ln \left(\bar{\tau}_{t}^{(i)}(s)-z\right) \theta(s) d s$,

$Q_{t}^{(i)}(z)=\int q_{t}^{(i)}(z) d z=\frac{-i}{2 k_{t}^{(i)}} \int_{\Gamma_{i}} f^{*}\left(\tau_{t}^{(i)}(s)-z\right) h_{n}(s) d s$

$+\frac{1}{2} \int_{\Gamma_{i}}\left(n_{2}(s)-p_{t}^{(i)} n_{1}(s)\right) \ln \left(\tau_{t}^{(i)}(s)-z\right) \theta(s) d s$,

$f^{*}(z)=z(\ln (z)-1), K=\left(k_{t}^{(1)}-k_{t}^{(2)}\right) /\left(k_{t}^{(1)}+k_{t}^{(2)}\right)$.

\subsection{Thermoelasticity of an anisotropic bimaterial}

For obtaining the integral formulae of thermoelectroelasticity one should write the Cauchy integral formula (9) for the Stroh complex vector functions $f^{(1)}\left(z_{*}^{(1)}\right)$ and $f^{(2)}\left(z_{*}^{(2)}\right)$, which are analytic in the domains $S_{1}$ and $S_{2}$, respectively. Since the Cauchy integral formula define the analytic function that vanishes at the infinity, the complete solution of the problem can be presented as a sum of the perturbed solution defined by the Cauchy formula and a homogeneous solution given by the functions $f_{\infty}^{(1)}\left(z_{*}^{(1)}\right)$ and $f_{\infty}^{(2)}\left(z_{*}^{(2)}\right)$, which satisfy boundary conditions (8). Consequently, one obtains

$\mathbf{f}^{(1)}\left(z_{*}^{(1)}\right)=\mathbf{f}_{\infty}^{(1)}\left(z_{*}^{(1)}\right)+\frac{1}{2 \pi i}\left[\int_{\Gamma_{1}}\left\langle\frac{d \tau_{*}^{(1)}}{\tau_{*}^{(1)}-z_{*}^{(1)}}\right) \mathbf{f}^{(1)}\left(\tau_{*}^{(1)}\right)\right.$
$\left.+\int_{-\infty}^{+\infty}\left\langle\frac{d x_{1}}{x_{1}-z_{*}^{(1)}}\right) \mathbf{f}^{(1)}\left(x_{1}\right)\right] \quad\left(\operatorname{Im} z_{*}^{(1)}>0\right)$

$\operatorname{Im} z_{\beta}^{(1)}>0$ :

$\int_{\Gamma_{1}}\left\langle\frac{d \tau_{*}^{(1)}}{\tau_{*}^{(1)}-\bar{z}_{\beta}^{(1)}}\right\rangle \mathbf{f}^{(1)}\left(\tau_{*}^{(1)}\right)+\int_{-\infty}^{+\infty} \frac{d x_{1}}{x_{1}-\bar{z}_{\beta}^{(1)}} \mathbf{f}^{(1)}\left(x_{1}\right)=0$,

$\int_{\Gamma_{2}}\left\langle\frac{d \tau_{*}^{(2)}}{\tau_{*}^{(2)}-z_{\beta}^{(1)}}\right\rangle \mathbf{f}^{(2)}\left(\tau_{*}^{(2)}\right)-\int_{-\infty}^{+\infty} \frac{d x_{1}}{x_{1}-z_{\beta}^{(1)}} \mathbf{f}^{(2)}\left(x_{1}\right)$,

$\mathbf{f}^{(2)}\left(z_{*}^{(2)}\right)=\mathbf{f}_{\infty}^{(2)}\left(z_{*}^{(2)}\right)+\frac{1}{2 \pi i}\left[\int_{\Gamma_{2}}\left\langle\frac{d \tau_{*}^{(2)}}{\tau_{*}^{(2)}-z_{*}^{(2)}}\right\rangle \mathbf{f}^{(2)}\left(\tau_{*}^{(2)}\right)\right.$

$\left.-\int_{-\infty}^{+\infty}\left\langle\frac{d x_{1}}{x_{1}-z_{*}^{(2)}}\right) \mathbf{f}^{(2)}\left(x_{1}\right)\right] \quad\left(\operatorname{Im} z_{*}^{(2)}<0\right) ;$

$\operatorname{Im} z_{\beta}^{(2)}<0$ :

$\int_{\Gamma_{1}}\left\langle\frac{d \tau_{*}^{(1)}}{\tau_{*}^{(1)}-z_{\beta}^{(2)}}\right) \mathbf{f}^{(1)}\left(\tau_{*}^{(1)}\right)+\int_{-\infty}^{+\infty} \frac{d x_{1}}{x_{1}-z_{\beta}^{(2)}} \mathbf{f}^{(1)}\left(x_{1}\right)=0 ;$

$\int_{\Gamma_{2}}\left(\frac{d \tau_{*}^{(2)}}{\tau_{*}^{(2)}-\bar{z}_{\beta}^{(2)}}\right) \mathbf{f}^{(2)}\left(\tau_{*}^{(2)}\right)-\int_{-\infty}^{+\infty} \frac{d x_{1}}{x_{1}-\bar{z}_{\beta}^{(2)}} \mathbf{f}^{(2)}\left(x_{1}\right)$,

where: $\left\langle F\left(z_{*}\right)\right\rangle=\operatorname{diag}\left[F_{1}\left(z_{1}\right), F_{2}\left(z_{2}\right), F_{3}\left(z_{3}\right)\right]$,

$z_{\beta}^{(i)}=x_{1}+p_{\beta}^{(i)} x_{2}(\beta=1, \ldots, 3)$.

Utilizing Eqs (4) and (8), the improper integrals in Eqs (36)(41) can be rewritten as follows: where

$\mathbf{m}\left(z_{\beta}^{(j)}\right)=\int_{-\infty}^{+\infty} \frac{\phi\left(x_{1}\right) d x_{1}}{x_{1}-z_{\beta}^{(j)}}$,

$\mathbf{p}\left(z_{\beta}^{(j)}\right)=\int_{-\infty}^{+\infty} \frac{\mathbf{u}\left(x_{1}\right) d x_{1}}{x_{1}-z_{\beta}^{(j)}}$.

Integrating by parts the second term in Eq (42) and accounting for $\mathrm{Eq}(4)$, one can obtain that

$$
\begin{aligned}
& \int_{-\infty}^{+\infty} \frac{\mathbf{f}^{(1)} d x_{1}}{x_{1}-z_{\beta}^{(i)}}=\mathbf{A}_{1}^{\mathrm{T}} \mathbf{m}\left(z_{\beta}^{(i)}\right)+\mathbf{B}_{1}^{\mathrm{T}} \mathbf{p}\left(z_{\beta}^{(i)}\right)+\mu_{1} M_{t}\left(z_{\beta}^{(i)}\right) \\
& -\lambda_{1} P_{t}\left(z_{\beta}^{(i)}\right)+\rho_{0} \lambda_{1} m_{t}\left(z_{\beta}^{(i)}\right) \\
& \int_{-\infty}^{+\infty} \frac{\mathbf{f}^{(2)} d x_{1}}{x_{1}-z_{\beta}^{(i)}}=\mathbf{A}_{2}^{\mathrm{T}} \mathbf{m}\left(z_{\beta}^{(i)}\right)+\mathbf{B}_{2}^{\mathrm{T}} \mathbf{p}\left(z_{\beta}^{(i)}\right)+\mu_{2} M_{t}\left(z_{\beta}^{(i)}\right) \\
& -\lambda_{2} P_{t}\left(z_{\beta}^{(i)}\right)
\end{aligned}
$$

where the complex constants $\mu_{i}$ and $\lambda_{i}$ are defined as:

$\mu_{i}=\frac{1}{k_{t}^{(i)}}\left(\mathbf{A}_{i}^{\mathrm{T}} \operatorname{Im}\left[\mathbf{d}_{i}\right]+\mathbf{B}_{i}^{\mathrm{T}} \operatorname{Im}\left[\mathbf{c}_{i}\right]\right)$,

$\lambda_{i}=\mathbf{A}_{i}^{\mathrm{T}} \operatorname{Re}\left[\mathbf{d}_{i}\right]+\mathbf{B}_{i}^{\mathrm{T}} \operatorname{Re}\left[\mathbf{c}_{i}\right]$

Denoting the Cauchy integrals of the Stroh complex functions as:

$\mathbf{q}_{j}\left(z_{\beta}^{(i)}\right)=\int_{\Gamma_{j}}\left\langle\frac{d \tau_{*}^{(j)}}{\tau_{*}^{(j)}-z_{\beta}^{(i)}}\right\rangle \mathbf{f}^{(j)}\left(\tau_{*}^{(j)}\right)$
$\overline{\mathbf{q}}_{j}\left(z_{\beta}^{(i)}\right)=\int_{\Gamma_{j}}\left\langle\frac{d \bar{\tau}_{*}^{(j)}}{\overline{\bar{\tau}_{*}^{(j)}-z_{\beta}^{(i)}}}\right\rangle \overline{\mathbf{f}^{(j)}\left(\tau_{*}^{(j)}\right) .}$

Eqs (36)-(41) can be rewritten as follows:

$$
\begin{aligned}
& \mathbf{f}^{(1)}\left(z_{*}^{(1)}\right)=\mathbf{f}_{\infty}^{(1)}\left(z_{*}^{(1)}\right)+\frac{1}{2 \pi i}\left[\mathbf{q}_{1}\left(z_{*}^{(1)}\right)\right. \\
& +\sum_{\beta=1}^{3} \mathbf{I}_{\beta}\left(\mathbf{A}_{1}^{\mathrm{T}} \mathbf{m}\left(z_{\beta}^{(1)}\right)+\mathbf{B}_{1}^{\mathrm{T}} \mathbf{p}\left(z_{\beta}^{(1)}\right)\right) \\
& \left.+\left\langle M_{t}\left(z_{*}^{(1)}\right)\right| \mu_{1}-\left\langle P_{t}\left(z_{*}^{(1)}\right)\right| \lambda_{1}+\rho_{0}\left\langle m_{t}\left(z_{*}^{(1)}\right)\right| \lambda_{1}\right] ; \\
& \overline{\mathbf{q}}_{1}\left(z_{\beta}^{(1)}\right)+\overline{\mathbf{A}}_{1}^{\mathrm{T}} \mathbf{m}\left(z_{\beta}^{(1)}\right)+\overline{\mathbf{B}}_{1}^{\mathrm{T}} \mathbf{p}\left(z_{\beta}^{(1)}\right)+M_{t}\left(z_{\beta}^{(1)}\right) \bar{\mu}_{1} \\
& -P_{t}\left(z_{\beta}^{(1)}\right) \bar{\lambda}_{1}+\rho_{0} m_{t}\left(z_{\beta}^{(1)}\right) \bar{\lambda}_{1}=0 ; \\
& \mathbf{q}_{2}\left(z_{\beta}^{(1)}\right)-\mathbf{A}_{2}^{\mathrm{T}} \mathbf{m}\left(z_{\beta}^{(1)}\right)-\mathbf{B}_{2}^{\mathrm{T}} \mathbf{p}\left(z_{\beta}^{(1)}\right)-M_{t}\left(z_{\beta}^{(1)}\right) \mu_{2} \\
& +P_{t}\left(z_{\beta}^{(1)}\right) \lambda_{2}=0 ; \\
& \mathbf{f}^{(2)}\left(z_{*}^{(2)}\right)=\mathbf{f}_{\infty}^{(2)}\left(z_{*}^{(2)}\right)+\frac{1}{2 \pi i}\left[\mathbf{q}_{2}\left(z_{*}^{(2)}\right)\right. \\
& -\sum_{\beta=1}^{3} \mathbf{I}_{\beta}\left(\mathbf{A}_{2}^{\mathrm{T}} \mathbf{m}\left(z_{\beta}^{(2)}\right)+\mathbf{B}_{2}^{\mathrm{T}} \mathbf{p}\left(z_{\beta}^{(2)}\right)\right) \\
& \left.-\left\langle M_{t}\left(z_{*}^{(2)}\right)\right\rangle \mu_{2}+\left\langle P_{t}\left(z_{*}^{(2)}\right)\right| \lambda_{2}\right] ;
\end{aligned}
$$


$\mathbf{q}_{1}\left(z_{\beta}^{(2)}\right)+\mathbf{A}_{1}^{\mathrm{T}} \mathbf{m}\left(z_{\beta}^{(2)}\right)+\mathbf{B}_{1}^{\mathrm{T}} \mathbf{p}\left(z_{\beta}^{(2)}\right)+M_{t}\left(z_{\beta}^{(2)}\right) \mu_{1}$

$-P_{t}\left(z_{\beta}^{(2)}\right) \lambda_{1}+\rho_{0} m_{t}\left(z_{\beta}^{(2)}\right) \lambda_{1}=0$;

$\overline{\mathbf{q}}_{2}\left(z_{\beta}^{(2)}\right)-\overline{\mathbf{A}}_{2}^{\mathrm{T}} \mathbf{m}\left(z_{\beta}^{(2)}\right)-\overline{\mathbf{B}}_{2}^{\mathrm{T}} \mathbf{p}\left(z_{\beta}^{(2)}\right)-M_{t}\left(z_{\beta}^{(2)}\right) \bar{\mu}_{2}$

$+P_{t}\left(z_{\beta}^{(2)}\right) \bar{\lambda}_{2}=0$,

where: $\mathbf{I}_{1}=\operatorname{diag}[1,0,0], \mathbf{I}_{2}=\operatorname{diag}[0,1,0], \mathbf{I}_{3}=\operatorname{diag}[0,0,1]$.

Eqs (49), (50), (52), (53) allow to express the improper integrals (43) along the infinite path through the path integrals over the contours $\Gamma_{j}$ :

$$
\begin{aligned}
& \mathbf{m}\left(z_{\beta}^{(1)}\right)=\left(\overline{\mathbf{A}}_{1} \overline{\mathbf{B}}_{1}^{-1}-\mathbf{A}_{2} \mathbf{B}_{2}^{-1}\right)^{-\mathrm{T}}\left(\overline{\mathbf{B}}_{1}^{-\mathrm{T}} \mathbf{y}_{1}\left(z_{\beta}^{(1)}\right)\right. \\
& \left.\quad-\mathbf{B}_{2}^{-\mathrm{T}} \mathbf{y}_{2}\left(z_{\beta}^{(1)}\right)\right), \\
& \mathbf{p}\left(z_{\beta}^{(1)}\right)=\left(\overline{\mathbf{B}}_{1} \overline{\mathbf{A}}_{1}^{-1}-\mathbf{B}_{2} \mathbf{A}_{2}^{-1}\right)^{-\mathrm{T}}\left(\overline{\mathbf{A}}_{1}^{-\mathrm{T}} \mathbf{y}_{1}\left(z_{\beta}^{(1)}\right)-\right. \\
& \left.\mathbf{A}_{2}^{-\mathrm{T}} \mathbf{y}_{2}\left(z_{\beta}^{(1)}\right)\right), \\
& \mathbf{y}_{1}\left(z_{\beta}^{(1)}\right)=-\overline{\mathbf{q}}_{1}\left(z_{\beta}^{(1)}\right)-M_{t}\left(z_{\beta}^{(1)}\right) \bar{\mu}_{1}+P_{t}\left(z_{\beta}^{(1)}\right) \bar{\lambda}_{1} \\
& -\rho_{0} m_{t}\left(z_{\beta}^{(1)}\right) \bar{\lambda}_{1}, \\
& \mathbf{y}_{2}\left(z_{\beta}^{(1)}\right)=\mathbf{q}_{2}\left(z_{\beta}^{(1)}\right)-M_{t}\left(z_{\beta}^{(1)}\right) \mu_{2}+P_{t}\left(z_{\beta}^{(1)}\right) \lambda_{2}, \\
& \mathbf{m}\left(z_{\beta}^{(2)}\right)=\left(\overline{\mathbf{A}}_{2} \overline{\mathbf{B}}_{2}^{-1}-\mathbf{A}_{1} \mathbf{B}_{1}^{-1}\right)^{-\mathrm{T}}\left(\overline{\mathbf{B}}_{2}^{-\mathrm{T}} \mathbf{y}_{3}\left(z_{\beta}^{(2)}\right)\right. \\
& \mathbf{p}\left(z_{\beta}^{(2)}\right)=\left(\overline{\mathbf{B}}_{2} \overline{\mathbf{A}}_{2}^{-1}-\mathbf{B}_{4}\left(z_{\beta}^{(2)}\right)\right), \\
& \left.\mathbf{A}_{1}^{-\mathrm{T}} \mathbf{y}_{4}\left(z_{\beta}^{(2)}\right)\right), \\
& \mathbf{y}_{3}\left(z_{\beta}^{(2)}\right)=\overline{\mathbf{q}}_{2}\left(z_{\beta}^{(2)}\right)-M_{t}\left(z_{\beta}^{(2)}\right) \overline{\mathbf{A}}_{2}+P_{t}\left(z_{\beta}^{(2)}\right) \bar{\lambda}_{2}, \\
& \mathbf{y}_{4}\left(z_{\beta}^{(2)}\right)=-\mathbf{q}_{1}\left(z_{\beta}^{(2)}\right)-M_{t}\left(z_{\beta}^{(2)}\right) \mu_{1}+P_{t}\left(z_{\beta}^{(2)}\right) \lambda_{1} \\
& -\rho_{0} m_{t}\left(z_{\beta}^{(2)}\right) \lambda_{1} .
\end{aligned}
$$

Substituting Eqs (54), (55) into Eqs (48), (51), accounting for Eq (35), one can obtain the integral formulae for the Stroh complex vector-functions of thermoelastic anisotropic bimaterial with thermally imperfect interface, which do not contain the improper integrals over the infinite path (bimaterial interface):

$$
\begin{aligned}
& \mathbf{f}^{(1)}\left(z_{*}^{(1)}\right)=\mathbf{f}_{\infty}^{(1)}\left(z_{*}^{(1)}\right)+\frac{1}{2 \pi i}\left[\mathbf{q}_{1}\left(z_{*}^{(1)}\right)\right. \\
& +\sum_{\beta=1}^{3} \mathbf{I}_{\beta}\left(\mathbf{G}_{1}^{(1)} \overline{\mathbf{q}}_{1}\left(z_{\beta}^{(1)}\right)+\mathbf{G}_{2}^{(1)} \mathbf{q}_{2}\left(z_{\beta}^{(1)}\right)\right) \\
& +\left\langle\bar{Q}_{t}^{(1)}\left(z_{*}^{(1)}\right)\right\rangle \delta_{1}^{(1)}+\left\langle Q_{t}^{(2)}\left(z_{*}^{(1)}\right)\right\rangle \delta_{2}^{(1)} \\
& \left.+\left\langle e_{t}^{(2)}\left(z_{*}^{(1)}\right)+\bar{e}_{t}^{(1)}\left(z_{*}^{(1)}\right)\right\rangle \kappa^{(1)}\right], \\
& \mathbf{f}^{(2)}\left(z_{*}^{(2)}\right)=\mathbf{f}_{\infty}^{(2)}\left(z_{*}^{(2)}\right)+\frac{1}{2 \pi i}\left[\mathbf{q}_{2}\left(z_{*}^{(2)}\right)\right. \\
& -\sum_{\beta=1}^{3} \mathbf{I}_{\beta}\left(\mathbf{G}_{1}^{(2)} \mathbf{q}_{1}\left(z_{\beta}^{(2)}\right)+\mathbf{G}_{2}^{(2)} \overline{\mathbf{q}}_{2}\left(z_{\beta}^{(2)}\right)\right) \\
& +\left\langle Q_{t}^{(1)}\left(z_{*}^{(2)}\right)\right\rangle \delta_{1}^{(2)}+\left\langle\bar{Q}_{t}^{(2)}\left(z_{*}^{(2)}\right)\right\rangle \delta_{2}^{(2)} \\
& \left.+\left\langle e_{t}^{(1)}\left(z_{*}^{(2)}\right)+\bar{e}_{t}^{(2)}\left(z_{*}^{(2)}\right)\right\rangle \kappa^{(2)}\right],
\end{aligned}
$$

where the constants $\mathbf{G}_{i}^{(j)}$ and $\delta_{i}^{(j)}$ are the same as in Ref (Pas- ternak et al., 2014), and

$\kappa^{(1)}=-\delta_{2}^{(1)}+2 \mathbf{G}_{2}^{(1)} \lambda_{2} ; \kappa^{(2)}=-\delta_{1}^{(2)}-2 \mathbf{G}_{1}^{(2)} \lambda_{1}$.

Derived equations (56) and (57) for the Stroh complex functions allow writing the integral formulae relating the latter at the arbitrary point of the bimaterial solid with the boundary values of temperature, heat flux, displacement and traction at the contours $\Gamma_{i}$. Therefore, using Eqs (3), (4), (56), (57) and (Pasternak, 2012, Ting, 1996) one can obtain the following extended Somigliana integral identity for a thermoelastic bimaterial solid with imperfect thermal contact at its interface:

$\mathbf{u}(\xi)=\mathbf{u}^{\infty}(\xi)+\int_{\Gamma} \mathbf{U}^{\mathrm{bm}}(\mathbf{x}, \xi) \mathbf{t}(\mathbf{x}) d s(\mathbf{x})-$ $\int_{\Gamma} \mathbf{T}^{\mathrm{bm}}(\mathbf{x}, \xi) \mathbf{u}(\mathbf{x}) d s(\mathbf{x})+\int_{\Gamma} \mathbf{r}^{\mathrm{ITC}}(\mathbf{x}, \xi) \theta(\mathbf{x}) d s(\mathbf{x})+$ $\int_{\Gamma} \mathbf{v}^{\mathrm{ITC}}(\mathbf{x}, \xi) h_{n}(\mathbf{x}) d s(\mathbf{x})$,

$\sigma_{j}(\xi)=\sigma_{j}^{\infty}(\xi)+\int_{\Gamma} \mathbf{D}_{j}^{\mathrm{bm}}(\mathbf{x}, \xi) \mathbf{t}(\mathbf{x}) d s(\mathbf{x})-$

$\int_{\Gamma} \mathbf{s}_{j}^{\mathrm{bm}}(\mathbf{x}, \xi) \mathbf{u}(\mathbf{x}) d s(\mathbf{x})+\int_{\Gamma} \mathbf{q}_{j}^{\mathrm{ITC}}(\mathbf{x}, \xi) \theta(\mathbf{x}) d s(\mathbf{x})+$ $\int_{\Gamma} \mathbf{w}_{j}^{\mathrm{ITC}}(\mathbf{x}, \xi) h_{n}(\mathbf{x}) d s(\mathbf{x})$,

where: the kernels $\mathbf{U}^{\mathrm{bm}}(\mathbf{x}, \xi), \mathbf{T}^{\mathrm{bm}}(\mathbf{x}, \xi), \mathbf{D}_{j}^{\mathrm{bm}}(\mathbf{x}, \xi), \mathbf{S}_{j}^{\mathrm{bm}}(\mathbf{x}, \xi)$ are the same as those derived by Pasternak et al. (2014) for a thermoelectroelastic bimaterial with perfect thermal and mechanical interface, and others kernels are defined by the following equations:

$\mathbf{x} \in S_{1} \wedge \xi \in S_{1}:$

$\mathbf{r}^{I T C}(\mathbf{x}, \xi)=-\frac{1}{\pi} \operatorname{Im}\left\{\mathbf{A}_{1}\left(\left\langle\ln W_{*}^{(1,1)}\right\rangle \mathbf{n}^{(1)}\right.\right.$

$+\sum_{\beta=1}^{3}\left\langle\ln \bar{W}_{\beta}^{(1,1)}\right\rangle \mathbf{G}_{1}^{(1)} \mathbf{I}_{\beta} \overline{\mathbf{n}}^{(1)}-\frac{\bar{n}_{t}^{(1)}}{2}\left(\left\langle\ln \bar{W}_{t *}^{(1,1)}\right\rangle \delta_{1}^{(1)}\right.$

$\left.\left.-\left\langle\mathrm{K}\left(B^{(1)} \bar{W}_{t *}^{(1,1)}\right)\right) \mathbf{k}^{(1)}\right)\right)+\frac{\mathbf{c}_{1}}{2}(K-1) \bar{n}_{t}^{(1)} \mathrm{L}^{(1)}\left(\bar{W}_{t}^{(1,1)}\right)$

$\left.-\frac{\mathbf{c}_{1}}{2}\left(n_{t}^{(1)} \ln W_{t}^{(1,1)}-\bar{n}_{t}^{(1)} \ln \bar{W}_{t}^{(1,1)}\right)\right\}$,

$\mathbf{v}^{I T C}(\mathbf{x}, \xi)=\frac{1}{\pi} \operatorname{Im}\left\{\mathbf{A}_{1}\left[-\left\langle f^{*}\left(W_{*}^{(1,1)}\right)\right\rangle \mu_{1}\right.\right.$

$-\sum_{\beta=1}^{3}\left\langle f^{*}\left(\bar{W}_{\beta}^{(1,1)}\right)\right\rangle \mathbf{G}_{1}^{(1)} \mathbf{I}_{\beta} \bar{\mu}_{1}+\frac{i}{2 k_{t}^{(1)}}\left\langle f^{*}\left(\bar{W}_{t *}^{(1,1)}\right)\right\rangle \delta_{1}^{(1)}$

$\left.-\frac{\rho_{0}(1-K)}{4} \mathrm{~L}^{(1)}\left(\bar{W}_{t *}^{(1,1)}\right) \kappa^{(1)}\right]-\frac{i \mathbf{c}_{1}}{2 k_{t}^{(1)}}\left[f^{*}\left(W_{t}^{(1,1)}\right)\right.$

$\left.\left.+K f^{*}\left(\bar{W}_{t}^{(1,1)}\right)\right]+\frac{\rho_{0} \mathbf{c}_{1}(K-1)^{2}}{4} \mathrm{~L}^{(1)}\left(\bar{W}_{t}^{(1,1)}\right)\right\}$,

$\mathbf{q}_{j}^{I T C}(\mathbf{x}, \xi)=\frac{1}{\pi} \operatorname{Im}\left\{\mathbf{B}_{1} \delta_{*}^{(1)}\left[\left\langle\frac{1}{W_{*}^{(1,1)}}\right\rangle \mathbf{n}^{(1)}\right.\right.$

$+\sum_{\beta=1}^{3}\left\langle\frac{1}{\bar{W}_{\beta}^{(1,1)}}\right) \mathbf{G}_{1}^{(1)} \mathbf{I}_{\beta} \overline{\mathbf{n}}^{(1)}-\frac{1}{2} \bar{n}_{t}^{(1)}\left(\left\langle\frac{1}{\bar{W}_{t *}^{(1,1)}}\right)\left(\delta_{1}^{(1)}+\kappa^{(1)}\right)\right.$

$\left.\left.-B^{(1)}\left\langle K\left(B^{(1)} \bar{W}_{t *}^{(1,1)}\right)\right| \kappa^{(1)}\right)\right]$

$\left.-\frac{\mathbf{d}_{1}}{2} \delta_{t}^{(1)}\left(\left[\frac{n_{t}^{(1)}}{W_{t}^{(1,1)}}-\frac{\bar{n}_{t}^{(1)}}{\bar{W}_{t}^{(1,1)}}\right]+\frac{2 i \bar{n}_{t}^{(1)}}{\rho_{0} k_{t}^{(1)}} K\left(B^{(1)} \bar{W}_{t}^{(1,1)}\right)\right)\right\}$,

$\mathbf{w}_{j}^{I T C}(\mathbf{x}, \xi)=\frac{1}{\pi} \operatorname{Im}\left\{\mathbf{B}_{1} \delta_{*}^{(1)}\left[\left\langle\ln W_{*}^{(1,1)}\right\rangle \mu_{1}\right.\right.$

$+\sum_{\beta=1}^{3}\left\langle\ln \bar{W}_{\beta}^{(1,1)}\right\rangle \mathbf{G}_{1}^{(1)} \mathbf{I}_{\beta} \bar{\mu}_{1}-\frac{i}{2 k_{t}^{(1)}}\left(\left\langle\ln \bar{W}_{t *}^{(1,1)}\right\rangle \delta_{1}^{(1)}\right.$

$\left.\left.-\left\langle\mathrm{K}\left(B^{(1)} \bar{W}_{t *}^{(1,1)}\right)\right\rangle \kappa^{(1)}\right)\right]$

$+\frac{i \mathbf{d}_{1}}{2 k_{t}^{(1)}} \delta_{t}^{(1)}\left(\ln W_{t}^{(1,1)}+\ln \bar{W}_{t}^{(1,1)}+(K-1) \mathrm{L}^{(1)}\left(\bar{W}_{t}^{(1,1)}\right)\right\} ;$ 
$\mathbf{x} \in S_{2} \wedge \xi \in S_{1}:$

$\mathbf{r}^{I T C}(\mathbf{x}, \xi)=-\frac{1}{\pi} \operatorname{Im}\left\{\mathbf{A}_{1}\left[\sum_{\beta=1}^{3}\left\langle\ln W_{\beta}^{(2,1)}\right\rangle \mathbf{G}_{2}^{(1)} \mathbf{I}_{\beta} \mathbf{n}^{(2)}\right.\right.$

$\left.-\frac{1}{2} n_{t}^{(2)}\left(\left\langle\ln W_{t *}^{(2,1)}\right\rangle \delta_{2}^{(1)}\right)-\left\langle\mathrm{K}\left(B^{(1)} W_{t *}^{(2,1)}\right)\right\rangle \kappa^{(1)}\right]$

$\left.+\frac{\mathbf{c}_{1}}{2}(K-1) n_{t}^{(2)} \mathrm{L}^{(1)}\left(W_{t}^{(2,1)}\right)\right\}$,

$\mathbf{v}^{I T C}(\mathbf{x}, \xi)=-\frac{1}{\pi} \operatorname{Im}\left\{\mathbf{A}_{1}\left[\sum_{\beta=1}^{3}\left\langle f^{*}\left(W_{\beta}^{(2,1)}\right)\right\rangle \mathbf{G}_{2}^{(1)} \mathbf{I}_{\beta} \mu_{2}\right.\right.$

$\left.+\frac{i}{2 k_{t}^{(2)}}\left\langle f^{*}\left(W_{t *}^{(2,1)}\right)\right\rangle \delta_{2}^{(1)}-\frac{\rho_{0}(1+K)}{4} \mathrm{~L}^{(1)}\left(W_{t *}^{(2,1)}\right) \kappa^{(1)}\right]$

$\left.+\frac{i \mathbf{c}_{1}(1-K)}{2 k_{t}^{(2)}} f^{*}\left(W_{t}^{(2,1)}\right)-\frac{\rho_{0} \mathbf{c}_{1}\left(K^{2}-1\right)}{4} \mathrm{~L}^{(1)}\left(W_{t}^{(2,1)}\right)\right\}$,

$\mathbf{q}_{j}^{I T C}(\mathbf{x}, \xi)=\frac{1}{\pi} \operatorname{Im}\left\{\mathbf{B}_{1} \delta_{*}^{(1)}\left[\sum_{\beta=1}^{3}\left\langle\frac{1}{W_{\beta}^{(2,1)}}\right) \mathbf{G}_{2}^{(1)} \mathbf{I}_{\beta} \mathbf{n}^{(2)}\right.\right.$

$\left.-\frac{1}{2} n_{t}^{(2)}\left(\left|\frac{1}{W_{t *}^{(2,1)}}\right\rangle\left(\delta_{2}^{(1)}+\kappa^{(1)}\right)-B^{(1)}\left\langle\mathrm{K}\left(B^{(1)} W_{t *}^{(2,1)}\right)\right| \kappa^{(1)}\right)\right]$

$\left.-\frac{i \mathbf{d}_{1}}{\rho_{0} k_{t}^{(1)}} n_{t}^{(2)} \delta_{t}^{(1)} \mathrm{K}\left(B^{(1)} W_{t}^{(2,1)}\right)\right\}$

$\mathbf{w}_{j}^{I T C}(\mathbf{x}, \xi)=\frac{1}{\pi} \operatorname{Im}\left\{\mathbf{B}_{1} \delta_{*}^{(1)}\left[\sum_{\beta=1}^{3}\left\langle\ln W_{\beta}^{(2,1)}\right\rangle \mathbf{G}_{2}^{(1)} \mathbf{I}_{\beta} \mu_{2}\right.\right.$

$\left.+\frac{i}{2 k_{t}^{(2)}}\left(\left\langle\ln W_{t *}^{(2,1)}\right\rangle \delta_{2}^{(1)}-\left\langle\mathrm{K}\left(B^{(1)} W_{t *}^{(2,1)}\right)\right\rangle \kappa^{(1)}\right)\right]$

$\left.+\frac{i \mathbf{d}_{1}}{k_{t}^{(1)}+k_{t}^{(2)}} \delta_{t}^{(1)} \mathrm{L}^{(1)}\left(W_{t}^{(2,1)}\right)\right\}$

$\mathbf{x} \in S_{1} \wedge \xi \in S_{2}$ :

$\mathbf{r}^{I T C}(\mathbf{x}, \xi)=\frac{1}{\pi} \operatorname{Im}\left\{\mathbf{A}_{2}\left[\sum_{\beta=1}^{3}\left\langle\ln W_{\beta}^{(1,2)}\right\rangle \mathbf{G}_{1}^{(2)} \mathbf{I}_{\beta} \mathbf{n}^{(2)}\right.\right.$

$\left.+\frac{1}{2} n_{t}^{(1)}\left(\left\langle\ln W_{t *}^{(1,2)}\right| \delta_{1}^{(2)}-\left\langle\mathrm{K}\left(B^{(1)} W_{t *}^{(1,2)}\right)\right| \kappa^{(2)}\right)\right]$

$\left.+\frac{\mathbf{c}_{2}}{2}(K+1) n_{t}^{(1)} \mathrm{L}^{(2)}\left(W_{t}^{(1,2)}\right)\right\}$,

$\mathbf{v}^{I T C}(\mathbf{x}, \xi)=\frac{1}{\pi} \operatorname{Im}\left\{\mathbf{A}_{1}\left[\sum_{\beta=1}^{3}\left\langle f^{*}\left(W_{\beta}^{(1,2)}\right)\right\rangle \mathbf{G}_{1}^{(2)} \mathbf{I}_{\beta} \mu_{1}\right.\right.$

$\left.-\frac{i}{2 k_{t}^{(1)}}\left\langle f^{*}\left(W_{t *}^{(1,2)}\right)\right\rangle \delta_{1}^{(2)}-\frac{\rho_{0}(1-K)}{4} \mathrm{~L}^{(2)}\left(W_{t *}^{(1,2)}\right) \kappa^{(2)}\right]$

$\left.-\frac{i \mathbf{c}_{2}}{2 k_{t}^{(1)}}(1+K) f^{*}\left(W_{t}^{(1,2)}\right)-\frac{\rho_{0} \mathbf{c}_{2}\left(K^{2}-1\right)}{4} \mathrm{~L}^{(2)}\left(W_{t}^{(1,2)}\right)\right\}$,

$\mathbf{q}_{j}^{I T C}(\mathbf{x}, \xi)=-\frac{1}{\pi} \operatorname{Im}\left\{\mathbf{B}_{2} \delta_{*}^{(2)}\left[\sum_{\beta=1}^{3}\left|\frac{1}{W_{\beta}^{(1,2)}}\right\rangle \mathbf{G}_{1}^{(2)} \mathbf{I}_{\beta} \mathbf{n}^{(1)}\right.\right.$

$+\frac{1}{2} n_{t}^{(1)}\left(\mid \frac{1}{W_{t *}^{(1,2)}}\right)\left(\delta_{1}^{(2)}+\kappa^{(2)}\right)$

$\left.\left.+B^{(2)}\left\langle\mathrm{K}\left(B^{(2)} W_{t *}^{(1,2)}\right)\right| \kappa^{(2)}\right)\right]$

$\left.-\frac{i \mathbf{d}_{2}}{\rho_{0} k_{t}^{(2)}} n_{t}^{(1)} \delta_{t}^{(2)} \mathrm{K}\left(B^{(2)} W_{t}^{(1,2)}\right)\right\}$,

$\mathbf{w}_{j}^{I T C}(\mathbf{x}, \xi)=-\frac{1}{\pi} \operatorname{Im}\left\{\mathbf{B}_{2} \delta_{*}^{(2)}\left[\sum_{\beta=1}^{3}\left\langle\ln W_{\beta}^{(1,2)}\right\rangle \mathbf{G}_{1}^{(2)} \mathbf{I}_{\beta} \mu_{1}\right.\right.$

$\left.-\frac{i}{2 k_{t}^{(1)}}\left(\left\langle\ln W_{t *}^{(1,2)}\right\rangle \delta_{1}^{(2)}-\left\langle\mathrm{K}\left(B^{(2)} W_{t *}^{(1,2)}\right)\right\rangle \kappa^{(2)}\right)\right]$

$\left.-\frac{i \mathbf{d}_{2}}{k_{t}^{(1)}+k_{t}^{(2)}} \delta_{t}^{(2)} \mathrm{L}^{(2)}\left(W_{t}^{(1,2)}\right)\right\}$;

$\mathbf{x} \in S_{2} \wedge \xi \in S_{2}:$

$$
\begin{aligned}
& \mathbf{r}^{I T C}(\mathbf{x}, \xi)=-\frac{1}{\pi} \operatorname{Im}\left\{\mathbf { A } _ { 2 } \left(\left\langle\ln W_{*}^{(2,2)}\right\rangle \mathbf{n}^{(2)}\right.\right. \\
& -\sum_{\beta=1}^{3}\left\langle\ln \bar{W}_{\beta}^{(2,2)}\right\rangle \mathbf{G}_{2}^{(2)} \mathbf{I}_{\beta} \overline{\mathbf{n}}^{(2)}-\frac{1}{2} \bar{n}_{t}^{(2)}\left(\left\langle\ln \bar{W}_{t *}^{(2,2)}\right\rangle \delta_{2}^{(1)}\right. \\
& \left.\left.-\left\langle\mathrm{K}\left(B^{(2)} \bar{W}_{t *}^{(2,2)}\right)\right\rangle \kappa^{(2)}\right)\right)-\frac{\mathbf{c}_{2}}{2} \bar{n}_{t}^{(2)} \mathrm{L}^{(2)}\left(\bar{W}_{t}^{(2,2)}\right) \\
& \left.-\frac{\mathbf{c}_{2}}{2}\left(n_{t}^{(2)} \ln W_{t}^{(2,2)}-\bar{n}_{t}^{(2)} \ln \bar{W}_{t}^{(2,2)}\right)\right\} \text {, } \\
& \mathbf{v}^{I T C}(\mathbf{x}, \xi)=\frac{1}{\pi} \operatorname{Im}\left\{\mathbf { A } _ { 2 } \left[-\left\langle f^{*}\left(W_{*}^{(2,2)}\right)\right\rangle \mu_{2}\right.\right. \\
& +\sum_{\beta=1}^{3}\left\langle f^{*}\left(\bar{W}_{\beta}^{(2,2)}\right)\right\rangle \mathbf{G}_{2}^{(2)} \mathbf{I}_{\beta} \bar{\mu}_{2} \\
& \left.+\frac{i}{2 k_{t}^{(2)}}\left\langle f^{*}\left(\bar{W}_{t *}^{(2,2)}\right)\right\rangle \delta_{2}^{(2)}+\frac{\rho_{0}(1+K)}{4} \mathrm{~L}^{(2)}\left(\bar{W}_{t *}^{(2,2)}\right) \kappa^{(2)}\right] \\
& -\frac{i \mathbf{c}_{2}}{2 k_{t}^{(2)}}\left[f^{*}\left(W_{t}^{(2,2)}\right)-K f^{*}\left(\bar{W}_{t}^{(2,2)}\right)\right] \\
& \left.-\frac{\rho_{0} \mathbf{c}_{2}(K+1)^{2}}{4} \mathrm{~L}^{(2)}\left(\bar{W}_{t}^{(2,2)}\right)\right\} \text {, } \\
& \mathbf{q}_{j}^{I T C}(\mathbf{x}, \xi)=\frac{1}{\pi} \operatorname{Im}\left\{\mathbf { B } _ { 2 } \delta _ { * } ^ { ( 2 ) } \left[\left\langle\frac{1}{W_{*}^{(2,2)}}\right\rangle \mathbf{n}^{(2)}\right.\right. \\
& -\sum_{\beta=1}^{3}\left\langle\frac{1}{\bar{W}_{\beta}^{(2,2)}}\right\rangle \mathbf{G}_{2}^{(2)} \mathbf{I}_{\beta} \overline{\mathbf{n}}^{(2)}-\frac{1}{2} \bar{n}_{t}^{(2)}\left(\mid \frac{1}{\bar{W}_{t *}^{(2,2)}}\right)\left(\delta_{1}^{(2)}+\kappa^{(2)}\right) \\
& \left.\left.+B^{(2)}\left\langle\mathrm{K}\left(B^{(2)} \bar{W}_{t *}^{(2,2)}\right)\right| \kappa^{(2)}\right)\right] \\
& \left.-\frac{\mathbf{d}_{2}}{2} \delta_{t}^{(2)}\left(\left[\frac{n_{t}^{(2)}}{W_{t}^{(2,2)}}-\frac{\bar{n}_{t}^{(2)}}{\bar{W}_{t}^{(2,2)}}\right]-\frac{2 i}{\rho_{0} k_{t}^{(2)}} \bar{n}_{t}^{(2)} \mathrm{K}\left(B^{(2)} \bar{W}_{t}^{(2,2)}\right)\right)\right\}, \\
& \mathbf{w}_{j}^{I T C}(\mathbf{x}, \xi)=\frac{1}{\pi} \operatorname{Im}\left\{\mathbf { B } _ { 2 } \delta _ { * } ^ { ( 2 ) } \left[\left\langle\ln W_{*}^{(2,2)}\right\rangle \mu_{2}\right.\right. \\
& -\sum_{\beta=1}^{3}\left\langle\ln \bar{W}_{\beta}^{(2,2)}\right\rangle \mathbf{G}_{2}^{(2)} \mathbf{I}_{\beta} \bar{\mu}_{2}-\frac{i}{2 k_{t}^{(2)}}\left(\left\langle\ln \bar{W}_{t *}^{(2,2)}\right\rangle \delta_{2}^{(2)}\right. \\
& \left.\left.-\left\langle\mathrm{K}\left(B^{(2)} \bar{W}_{t *}^{(2,2)}\right)\right\rangle \kappa^{(2)}\right)\right] \\
& \left.+\frac{i \mathbf{d}_{2}}{2 k_{t}^{(2)}} \delta_{t}^{(2)}\left(\ln W_{t}^{(2,2)}-\ln \bar{W}_{t}^{(2,2)}-(1+K) \mathrm{L}^{(2)}\left(\bar{W}_{t}^{(2,2)}\right)\right)\right\} \text {. }
\end{aligned}
$$

The following constants and functions are used for contracting the notations:

$$
\begin{aligned}
& \rho_{j}=\mathbf{A}_{j}^{T} \operatorname{Re}\left[p_{t}^{(j)} \mathbf{d}_{j}\right]+\mathbf{B}_{j}^{T} \operatorname{Re}\left[p_{t}^{(j)} \mathbf{c}_{j}\right], \\
& \mathbf{n}^{(i)}=\lambda_{i} n_{2}(\mathbf{x})-\rho_{i} n_{1}(\mathbf{x}), Z_{*}^{(i)}(\mathbf{x})=x_{1}+p_{*}^{(i)} x_{2}, \\
& W_{*}^{(i, j)}=Z_{*}^{(i)}(\mathbf{x})-Z_{*}^{(j)}(\xi), W_{\beta}^{(i, j)}=Z_{\beta}^{(i)}(\mathbf{x})-Z_{*}^{(j)}(\xi), \\
& \bar{W}_{\beta}^{(i, j)}=\bar{Z}_{\beta}^{(i)}(\mathbf{x})-Z_{*}^{(j)}(\xi), W_{t *}^{(i, j)}=Z_{t}^{(i)}(\mathbf{x})-Z_{*}^{(j)}(\xi), \\
& \bar{W}_{t *}^{(i, j)}=\bar{Z}_{t}^{(i)}(\mathbf{x})-Z_{*}^{(j)}(\xi), \delta_{*}^{(j)}=\left\langle\delta_{i 2}-\delta_{i 1} p_{*}^{(j)}\right\rangle .
\end{aligned}
$$

The proposed complex variable approach allow not only to derive the boundary integral formulae for anisotropic thermoelasticity, but also to derive singular boundary integral equations for solving the boundary value problems for thermoelastic bimaterial solids with thermally imperfect interface. In particular, for derivation of the boundary integral equations it is convenient to apply the Sokhotskii-Plemelj formula (Muskhelishvili, 2008), which relates the limit value of the Cauchy integral over a smooth closed contour with its principal value. Thus, according to Eq (59) and (Muskhelishvili, 2008, Pasternak, 2012), for a smooth closed contours $\Gamma$ in a thermoelastic bimaterial one can obtain the following boundary integral equations for determination of the unknown boundary functions: 
$\frac{1}{2} \theta(\mathbf{y})=\theta^{\infty}(\mathbf{y})+\operatorname{RPV} \int_{\Gamma} \Theta^{I T C *}(\mathbf{x}, \mathbf{y}) h_{n}(\mathbf{x}) d s(\mathbf{x})-$ $\operatorname{CPV} \int_{\Gamma} H^{I T C *}(\mathbf{x}, \mathbf{y}) \theta(\mathbf{x}) d s(\mathbf{x})$,

$\frac{1}{2} \mathbf{u}(\mathbf{y})=\mathbf{u}^{\infty}(\xi)+\operatorname{RPV} \int_{\Gamma} \mathbf{U}^{\mathrm{bm}}(\mathbf{x}, \mathbf{y}) \mathbf{t}(\mathbf{x}) d s(\mathbf{x})-$

$\operatorname{CPV} \int_{\Gamma} \mathbf{T}^{\mathrm{bm}}(\mathbf{x}, \mathbf{y}) \mathbf{u}(\mathbf{x}) d s(\mathbf{x})+$

$\operatorname{RPV} \int_{\Gamma} \mathbf{r}^{I T C}(\mathbf{x}, \mathbf{y}) \theta(\mathbf{x}) d s(\mathbf{x})+$

$\int_{\Gamma} \mathbf{v}^{I T C}(\mathbf{x}, \mathbf{y}) h_{n}(\mathbf{x}) d s(\mathbf{x})$,

where RPV stands for a Riemann Principal Value and CPV for a Cauchy Principal Value of an integral.

The integral equations (65) degenerate, when separate closed contours $\Gamma_{j}$ of a line $\Gamma$ are the faces of mathematical cuts $\Gamma_{C j}$ (the simple opened arcs). In this case it is necessary to apply the theory of dual hypersingular integral equations (Pasternak et al., 2013a, Pasternak, 2012).

According to (Pasternak et al., 2013a), the stress and displacement discontinuity functions at the tips of thin inhomogeneities, which are not placed at the bimaterial interface, possess square root singularity. And the stress field in the vicinity of inclusion's tip is completely defined by the generalized stress intensity factors. The latter are related to the displacement and stress discontinuities at thin inhomogeneity with the following equations

$\mathbf{k}^{(1)}=\lim _{s \rightarrow 0} \sqrt{\frac{\pi}{8 s}} \mathbf{L} \cdot \Delta \mathbf{u}(s), \mathbf{k}^{(2)}=-\lim _{s \rightarrow 0} \sqrt{\frac{\pi s}{2}} \Sigma \mathbf{t}(s)$,

where: $\quad \mathbf{k}^{(1)}=\left[K_{21}, K_{11}, K_{31}\right]^{\mathrm{T}}, \quad \mathbf{k}^{(2)}=\left[K_{12}^{(2)}, K_{22}^{(2)}, K_{32}\right]^{\mathrm{T}}$; $K_{i j}$ are generalized stress intensity factors (SIF) (Pasternak et al, 2013b, Sulym, 2007); and $\boldsymbol{L}=-2 \sqrt{-1} \boldsymbol{B} \boldsymbol{B}^{T}$ is a real Barnett Lothe tensor (Sulym et al., 2014).

Obtained dual boundary integral equations along with the models of thin thermoelastic inclusions (Pasternak et al., 2013a) allow solving thermoelastic problems for a bimaterial solid with thermally imperfect interface, which components contain thin inhomogeneities.

\section{NUMERICAL EXAMPLES}

Similarly to (Pasternak et al., 2013a, Pasternak, 2012), obtained integral formulae and equations (25), (26), (59) are introduced entered into the computational algorithm of the modified boundary element method (Pasternak et al., 2013a, 2014) that allows numerical solution of a wide range of $2 \mathrm{D}$ problems for an anisotropic thermoelastic bimaterial solid with thermally imperfect interface containing internal inhomogeneities. Several numerical examples are considered here for a bimaterial containing thin thermoelastic inclusions. The boundary element mesh consists of only 20 discontinuous three-node boundary elements including two special for convenient determination of stress intensity factors at inclusion's tips.

Consider an anisotropic thermoelastic bimaterial (Fig. 2) consisting of two half-space that have identical mechanical and thermal properties. The bimaterial contains a thin rectilinear elastic isotropic inclusion of length $2 a$ and thickness $2 h=0.02 \mathrm{a}$ placed at the upper half-space $x_{2}>0$ parallel to the interface. The centre of the inclusion is placed at the distance $\mathrm{d}$ to the bimaterial interface. In the lower half-space $x_{2}<0$ at the distance $d / 2$ to the interface and at the distance a to the central vertical axis a heat source and a heat drain both of the same magnitude are placed. The half-spaces of the bimaterial possess the same thermal and mechanical properties of glass/epoxy: $E_{1}=55 \mathrm{GPa}$, $E_{2}=21 \mathrm{GPa}, \quad G_{12}=9.7 \mathrm{GPa}, \quad v_{12}=0.25, \quad \alpha_{11}=6.3$. $10^{-6} \mathrm{~K}-1, \quad \alpha_{22}=2.0 \cdot 10^{-5} \mathrm{~K}-1, \quad k_{11}=3.46 \mathrm{~W} /(\mathrm{m} \cdot \mathrm{K})$, $k_{22}=0.35 \mathrm{~W} /(\mathrm{m} \cdot \mathrm{K})$. Material symmetry axes coincide with the reference coordinates. Plane stress is considered.



Fig. 2. Bimaterial with identical properties of half-spaces
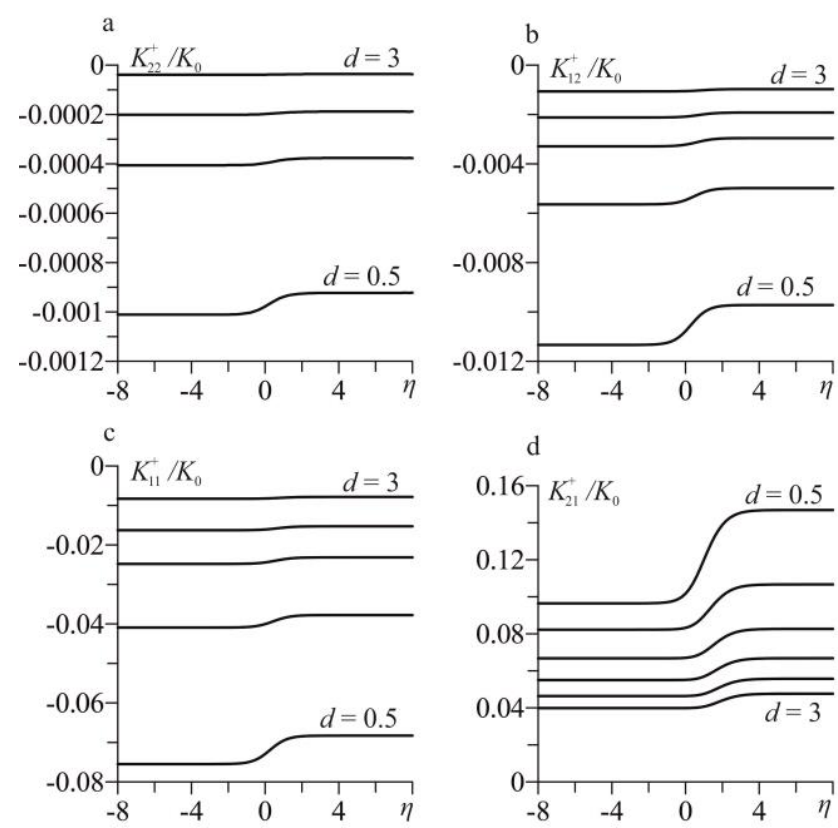

Fig. 3. Generalized stress intensity factors at the right tip of the inclusion, which relative rigidity is $10^{2}(\mathrm{a}-\mathrm{b})$ or $10^{-2}(\mathrm{c}-\mathrm{d})$

Fig. 3 presents the dependence of the generalized stress intensity factors (SIF) at the right tip of the inclusion on the parameter $\eta$, which define the thermal resistance of the interface as $\rho_{0}=$ $a / k_{11} \cdot 10^{\eta}$. The normalization factor is equal to $K_{0}=$ $\sqrt{\pi a} \cdot E_{1} \cdot \alpha_{11} / k_{11} \cdot q$. Plots are obtained for different values of the parameter $\mathrm{d}$ and the relative rigidity $k=G^{i} / G_{12}$ of the inclusion, where $\mathrm{G}^{\mathrm{i}}$ is a shear modulus of inclusion's material. It is assumed that the inclusion does not possess thermal expansion.

One can see in Fig. 3 that the closer are the heat sources and the inclusion to the bimaterial interface, the higher are the values of generalized SIF. Decrease in inclusion's rigidity considerably increases stress intensity. It should be mentioned that the 
most significant growth of all generalized SIF occurs, when the parameter $\eta$ of normalized thermal resistance of the bimaterial interface is near zero.
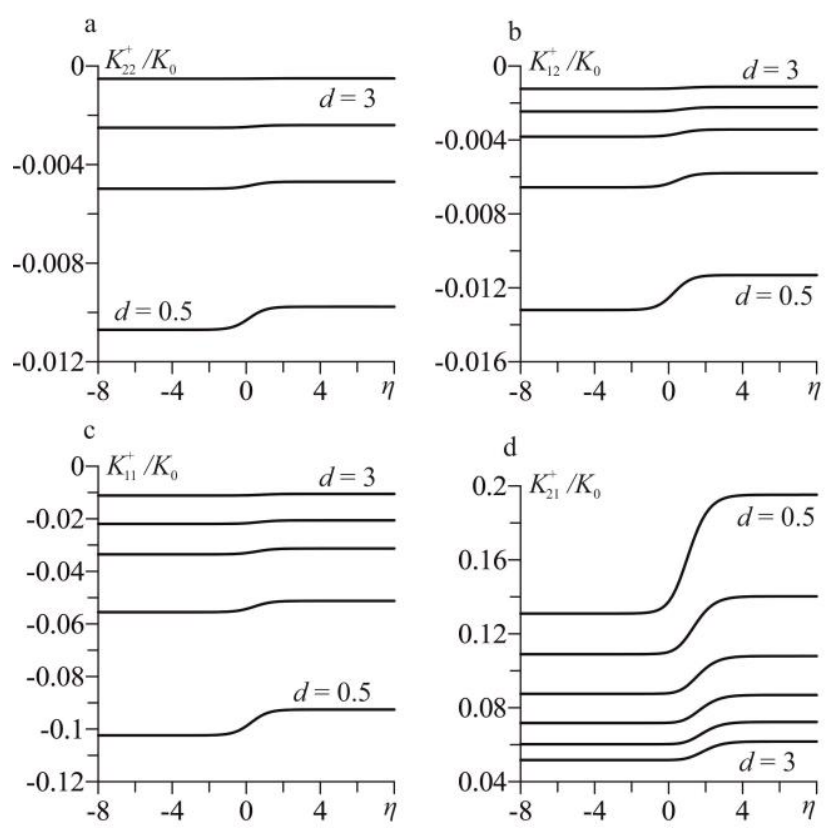

Fig. 4. Generalized stress intensity factors at the right tip of the inclusion, which relative rigidity is $10^{5}(\mathrm{a}-\mathrm{b})$ or $10^{-5}$ (c-d)

The increase in inclusion's relative rigidity (Fig. 4, a-b) causes a significant growth in the magnitude of the generalized SIF, and, in general, their behavior is the same, as well as in case of a softer inclusion. The same conclusion can be made, when relative rigidity of inclusion is equal to $10^{-5}$.

\section{CONCLUSION}

The paper presents a general complex variable straightforward approach for obtaining the boundary integral equations and integral formulae for a defective bimaterial solid with thermally imperfect interface. The kernels of these equations are obtained explicitly and in closed-form that allows developing the efficient boundary element approach for the analysis of thermoelastic anisotropic bimaterial solids with imperfect interface containing internal inhomogeneities. The influence of the thermal resistance of the interface on the field intensity factors at the tips of thin inhomogeneities is studied. It is shown that thermal resistance of the interface causes significant influence on the stress intensity at the tips of cracks even in the bimaterial consisting of two halfspaces with identical thermal and mechanical properties. Also several examples are considered for bimaterials with thin thermoelastic inclusions, and the intensity of heat flux and stress at their tips is studied.

\section{REFERENCES}

1. Benveniste Y. (2006), A general interface model for a threedimensional curved thin anisotropic interphase between two anisotropic media, J. Mech. Phys. Solids, 54, 708-734.

2. Chen T. (2001), Thermal conduction of a circular inclusion with variable interface parameter, Int. J. Solids. Struct., 38, 3081-3097.

3. Hwu C. (1992), Thermoelastic interface crack problems in dissimilar anisotropic media, Int. J. Solids. Struct., 18, 2077-2090.

4. Hwu C. (2010), Anisotropic elastic plates, Springer, London.

5. Jewtuszenko O., Adamowicz F., Grześ P., Kuciej M., Och E. (2014) Analytic and numerical modeling of the process of heat transfer in parts of disc of the brake systems, Publishing House of BUT (in Polish).

6. Kattis M. A., Mavroyannis G. (2006), Feeble interfaces in bimaterials, Acta Mech., 185, 11-29.

7. Muskhelishvili N.I. (2008), Singular integral equations, Dover publications, New York.

8. Pan E., Amadei B. (1999), Boundary element analysis of fracture mechanics in anisotropic bimaterials, Engineering Analysis with Boundary Elements, 23, 683-691.

9. Pasternak la. (2012), Boundary integral equations and the boundary element method for fracture mechanics analysis in 2D anisotropic thermoelasticity, Engineering Analysis with Boundary Elements, 36(12), 1931-1941.

10. Pasternak la., Pasternak R., Sulym H. (2013a), A comprehensive study on the 2D boundary element method for anisotropic thermoelectroelastic solids with cracks and thin inhomogeneities, Engineering Analysis with Boundary Elements, 37, №. 2, 419-433.

11. Pasternak la., Pasternak R., Sulym H. (2013b), Boundary integral equations for $2 \mathrm{D}$ thermoelasticity of a half-space with cracks and thin inclusions, Engineering Analysis with Boundary Elements, 37, 15141523.

12. Pasternak la., Pasternak R., Sulym H. (2014), Boundary integral equations and Green's functions for 2D thermoelectroelastic bimaterial, Engineering Analysis with Boundary Elements, 48, 87-101.

13. Qin Q.H. (2007), Green's function and boundary elements of multifield materials, Elsevier, Oxford.

14. Sulym H.T. (2007), Bases of mathematical theory of thermo-elastic equilibrium of solids containing thin inclusions, Research and Publishing center of NTSh, 2007 (in Ukrainian).

15. Sulym H.T., Pasternak la., Tomashivskyy M. (2014), Boundary element analysis of anisotropic thermoelastic half-space containing thin deformable inclusions, Ternopil Ivan Puluj National Technical University, 2014 (in Ukrainian).

16. Ting T.C.T. (1996), Anisotropic elasticity: theory and applications, Oxford University Press, New York.

17. Wang X., Pan E. (2010), Thermal Green's functions in plane anisotropic bimaterials with spring-type and Kapitza-type imperfect intrface, Acta Mech., 209, 115-128.

18. Yevtushenko A. A., Kuciej M. (2012), One-dimensional thermal problem of friction during braking: The history of development and actual state, International Journal of Heat and Mass Transfer, 55, $4148-4153$. 